\title{
Temporal variability of bidirectional seiching in an open-channel, lateral cavity
}

\author{
L. Engelen \\ Hydraulics Laboratory, Dept. of Civil Engineering, Ghent University, 9000 Ghent, Belgium \\ C. Perrot-Minot \\ Université de Lyon, LMFA, INSA Lyon, 69621 Villeurbanne, France \\ E. Mignot \\ Université de Lyon, LMFA, INSA Lyon, 69621 Villeurbanne, France \\ N. Rivière \\ Université de Lyon, LMFA, INSA Lyon, 69621 Villeurbanne, France \\ T. De Mulder \\ Hydraulics Laboratory, Dept. of Civil Engineering, Ghent University, 9000 Ghent, Belgium
}

\begin{abstract}
This paper presents laboratory results in which the free surface oscillations that are caused by the interaction between an open-channel flow and a lateral cavity are measured. For a square cavity with a side length equal to the width of the main stream, this oscillation phenomenon, named seiching, can result in standing waves in both the transverse and longitudinal direction for the same flow case. This bidirectional aspect of seiching is confirmed by the current results using a Fourier transform of the surface registrations in both the cavity and the main channel area. More importantly, a wavelet analysis in the time and frequency domains shows that bidirectional seiching is not a stationary phenomenon since the wavelet power of the two observed seiching modes varies significantly in time. Therefore, a more time-localised approach is used, which reveals that the entire measurement period can be subdivided in time windows where only one of the two seiching modes is considerably excited.
\end{abstract}

\section{INTRODUCTION}

The present study is dedicated to open-channel lateral cavities, i.e. semi-enclosed side-basins (e.g. cutoff meanders, ports, groyne fields, etc.) adjacent to a main stream (e.g. a fluvial or coastal current) flowing past their open end. The recirculating flow within the cavity exchanges mass and momentum with the main stream through the mixing layer that develops at the interface between the main channel and the cavity. This mixing layer is characterized by the advection of coherent turbulent structures that are periodically shed from the upstream corner and impinge on the downstream corner of the interface (Mignot et al. 2016). At this stagnation point, the impingement causes a fluctuating rise of the local water level and a corresponding periodic inflow towards the cavity.

Although free surface oscillations inside a lateral embayment may be generated by various (hydrodynamic or meteorological) excitation mechanisms, the current paper focuses on the seiching phenomenon in which the periodicity of the mixing layer is the main forcing mechanism. The resulting resonant gravity waves have a significant influence on the surface and flow conditions within the cavity (e.g. important for cargo handling inside a harbour basin), while Tuna et al. (2013) reported that the seiching may also increase mass exchange (e.g. sediments, pollutants or nutrients) between the main stream and the cavity up to $40 \%$.

Previous research (e.g. Kimura \& Hosoda 1997, Tuna et al. 2013, Mignot et al. 2015) suggested that seiching is triggered in case the natural frequency of the cavity is in agreement with the passing frequency of the vortices in the mixing layer, while Tuna et al. (2013) and Akutina (2015) found that 
also the flapping or 'jitter' phenomenon of the shear layer behaves in phase with the surface oscillations. For a square or rectangular cavity geometry, as is often studied in experimental research, it is possible to analytically compute the natural frequencies of the basin. Nonetheless, no method exists to predict the selected oscillation direction and the number of nodes characterising the standing gravity waves. Moreover, part of the literature only mentions a single type of standing wave, thus assuming a unidirectional sinusoidal surface shape. In contrast, Tsubaki \& Fujita (2006), Mignot et al. (2015) and Engelen et al. (2018) found that waves in both the transverse and longitudinal direction and/or several (higher-order) modes in a single oscillation direction may occur simultaneously.

In this work, we investigate the same geometry as Engelen et al. (2018), in which the width of the main channel and the width and length of the cavity are all three equal to each other (thus a square cavity). For such a particular geometry, bidirectional seiching is assumed to be promoted by the fact that some natural frequencies of transverse and longitudinal seiching are then equal or a multiple of each other. While a large number of flow cases was investigated in the context of this research, the current paper will focus on a single flow case and combine three simultaneous, pointwise surface registrations to estimate the 3D surface shape during the bidirectional seiching conditions. Moreover, a more detailed analysis in both the time and frequency domain is presented to investigate the stability of bidirectional seiching over time.

\section{EXPERIMENTAL SETUP}

The experiment described in this paper is conducted in the T-shaped confluence flume of the Hydraulics Laboratory of Ghent University (Fig. 1), of which both the main channel and the tributary have a width $b=0.40 \mathrm{~m}$. In the tributary, a water retaining wall is installed to create a lateral, square cavity with a length $L$ and width $W$ both equal to $0.40 \mathrm{~m}$ (thus $L=W=b$ ), and which is located $3 \mathrm{~m}$ downstream from the main channel inlet (total length of the flume equals $12 \mathrm{~m}$ ). The time evolution of the water depth in the cavity is measured using two pressure sensors located near the inner corners of the cavity $\left(P_{u}\right.$ and $\left.P_{d}\right)$, having an uncertainty of $\pm 0.05 \mathrm{~mm}$, while an ultrasonic gauge (uncertainty $<0.5$ $\mathrm{mm}$ ) measures the surface evolution in the main channel at a streamwise position that is equal to the downstream pressure sensor $(U)$. All three devices, of which the measurement positions are depicted in Figure 1, are synchronized at a sampling frequency of $50 \mathrm{~Hz}$ over a total measurement time of 90 minutes. The water depth $h_{m}$ is determined as the mean of the time-averaged water depths of the two pressure sensors and equals $14.5 \mathrm{~cm}$, thus $h_{m} / L=0.36$. The main stream discharge $Q$ is measured within the pumping loop of the flume using an electromagnetic flow meter, corresponding to a bulk main channel velocity $U_{b}=Q / b h_{m}=0.75 \mathrm{~m} / \mathrm{s}$. For the flow case discussed in this paper, this results in a Reynolds number $R e=U_{b} R / \nu=2.49 \times 10^{5}$ and a Froude number $F r=U_{b} / \sqrt{g h_{m}}=0.64$, in which $R=h_{m} /\left(b+2 h_{m}\right)$ denotes the hydraulic radius of the main channel, $\nu$ is the kinematic viscosity of water $\left(1.003 \times 10^{-6} \mathrm{~m}^{2} / \mathrm{s}\right.$ for water at $\left.20^{\circ} \mathrm{C}\right)$ and $g$ is the gravitational acceleration.

\section{THEORETICAL BACKGROUND OF SEICHING}

In a (semi-)enclosed basin, long-period standing surface oscillations may occur in case the forcing frequency equals the natural frequency of the basin. When such standing gravity waves are excited by the interaction with the main channel flow in (relatively shallow) harbours and lakes, they are often called seiches (Rabinovich 2010). In general, for a water body in a rectangular basin with a mean water depth $h_{m}$ and horizontal dimensions $L_{l}$ (longitudinal direction, noted $\mathrm{x}$ ) and $L_{t}$ (transverse direction, noted y), the natural frequencies $f_{n_{l} n_{t}}$ can theoretically be predicted by (Faltinsen et al. 2003):

$$
f_{n_{l} n_{t}}=\frac{1}{2 \pi} \sqrt{\pi g \sqrt{\left(\frac{n_{l}}{L_{l}}\right)^{2}+\left(\frac{n_{t}}{L_{t}}\right)^{2}} \tanh \left(\pi h_{m} \sqrt{\left(\frac{n_{l}}{L_{l}}\right)^{2}+\left(\frac{n_{t}}{L_{t}}\right)^{2}}\right)}
$$

in which $g$ denotes the gravitational acceleration and $n_{l}$ and $n_{t}$ indicate the number of nodes of the sinusoidal surface shape in respectively the longitudinal (x) and transverse (y) direction, such that both $n_{l}$ and $n_{t} \geq 0$ and $n_{l}+n_{t} \neq 0$. In the particular case of 1D seiching in either one of both directions, $n_{l}=0$ and $n_{t}>0$ (transverse seiching) or $n_{t}=0$ and $n_{l}>0$ (longitudinal seiching). In the following, 


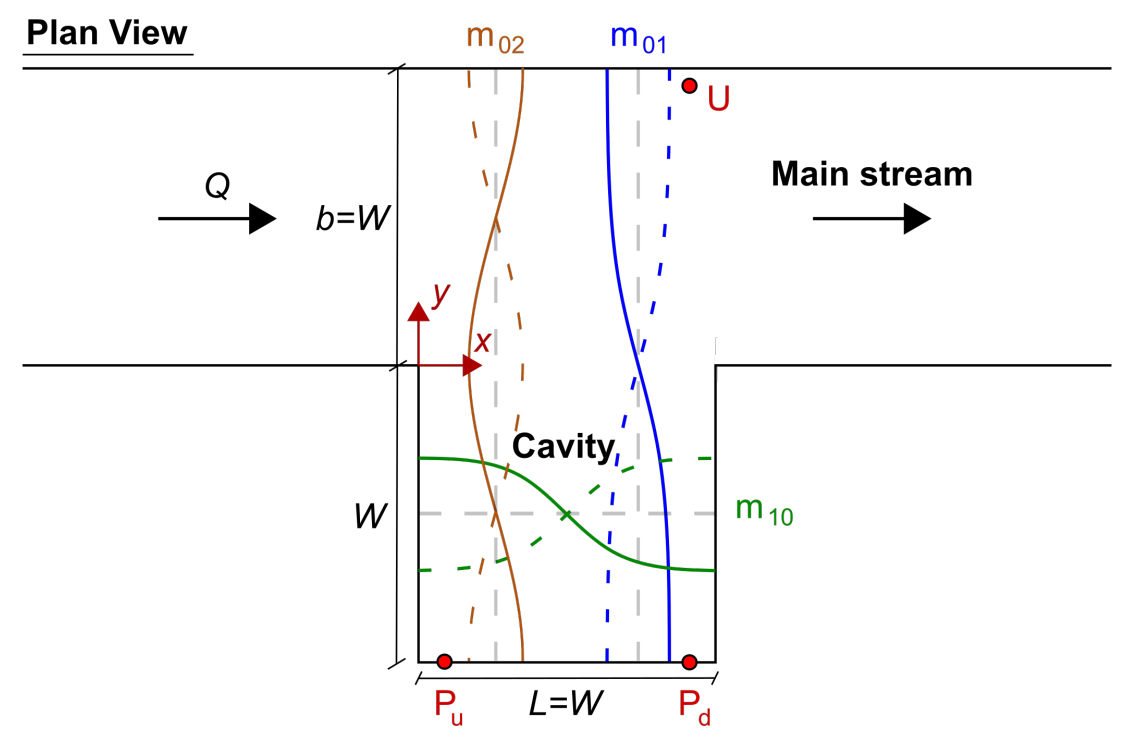

Figure 1. Schematic sketch of the experimental setup, indicating the location of the upstream $\left(P_{u}\right)$ and downstream $\left(P_{d}\right)$ pressure sensors in the water retaining wall at the closed end of the cavity, as well as the measurement location of the ultrasonic gauge $(U)$. The shape of the standing waves corresponding to the three seiching modes discussed in this paper (modes $m_{01}$ and $m_{02}$ in the y-direction and $m_{10}$ in the x-direction) are schematically displayed for the time instances at which they reach their maximum amplitude.

the different seiching modes will be distinguished based on the combination of $n_{l}$ and $n_{t}$ and will be denoted as $m_{n_{l} n_{t}}$ (Fig. 1).

Previous experiments on flow past a lateral cavity (Engelen et al. 2018) showed that bidirectional seiching may occur in the specific case where the streamwise length scale $L_{l}=L$ equals half of the crosswise length scale $L_{t}=b+W$, in which $b$ is the width of the main channel (along y-axis) and $L$ and $W$ are the length (along x-axis) and width (along y-axis) of the cavity, respectively (see Fig. 1). Substitution of $L_{t}=2 L_{l}$ in Equation 1 implies that the natural frequencies of unidirectional transverse seiching $\left(n_{t} \neq 0, n_{l}=0\right)$ equal half of the unidirectional longitudinal frequencies $\left(n_{t}=0, n_{l} \neq\right.$ 0 ). As a consequence, the pair modes of transverse seiching (with frequencies $f_{02}, f_{04}, f_{06}, \ldots$ ) have (theoretically) the same frequency as the longitudinal modes (with frequencies $f_{10}, f_{20}, f_{30}, \ldots$ ). For the flow case discussed in Section 4, Equation 1 predicts natural frequencies of $f_{01}=0.70 \mathrm{~Hz}$ and $f_{10}=f_{02}=1.26 \mathrm{~Hz}$.

\section{RESULTS}

Figure 2 gives the power spectral density ('Psd') of the surface oscillations, derived by a Fourier transform of the time series measured at $P_{u}, P_{d}$ and $U$. In the spectra of the pressure sensors $\left(P_{u}\right.$ and $\left.P_{d}\right)$, two peaks can be identified which approximately match the theoretical frequencies estimated with Equation 1 for $f_{01}$ and $f_{10}=f_{02}$. Since $U$ is located outside the region where longitudinal seiching is excited (the cavity), the absence of the peak at $f_{10}$ in the spectrum of the ultrasonic gauge confirms that this peak corresponds to longitudinal seiching $\left(m_{10}\right)$ and not to a higher order mode of transverse seiching $\left(m_{02}\right)$.

However, the spectra derived by the Fourier transform are acquired by integration over the entire acquisition time, such that information about the variability of the power-frequency distribution in time is lost. Therefore, in order to analyse the stability of seiching throughout the entire measurement period, this work employs a wavelet transform to derive the time-varying power spectrum of the seiching oscillations. In this work, the Continuous Wavelet Transform is applied using the complex Morlet wavelet as mother wavelet, the latter being characterised by the bandwidth parameter $\gamma_{b}$ (chosen equal to 5) and the wavelet centre frequency $\gamma_{c}$ (chosen equal to $1 \mathrm{~Hz}$ ). For more details about the wavelet transform, the reader is invited to refer to the literature (Torrence \& Compo 1998, Teolis \& Benedetto 1998). 


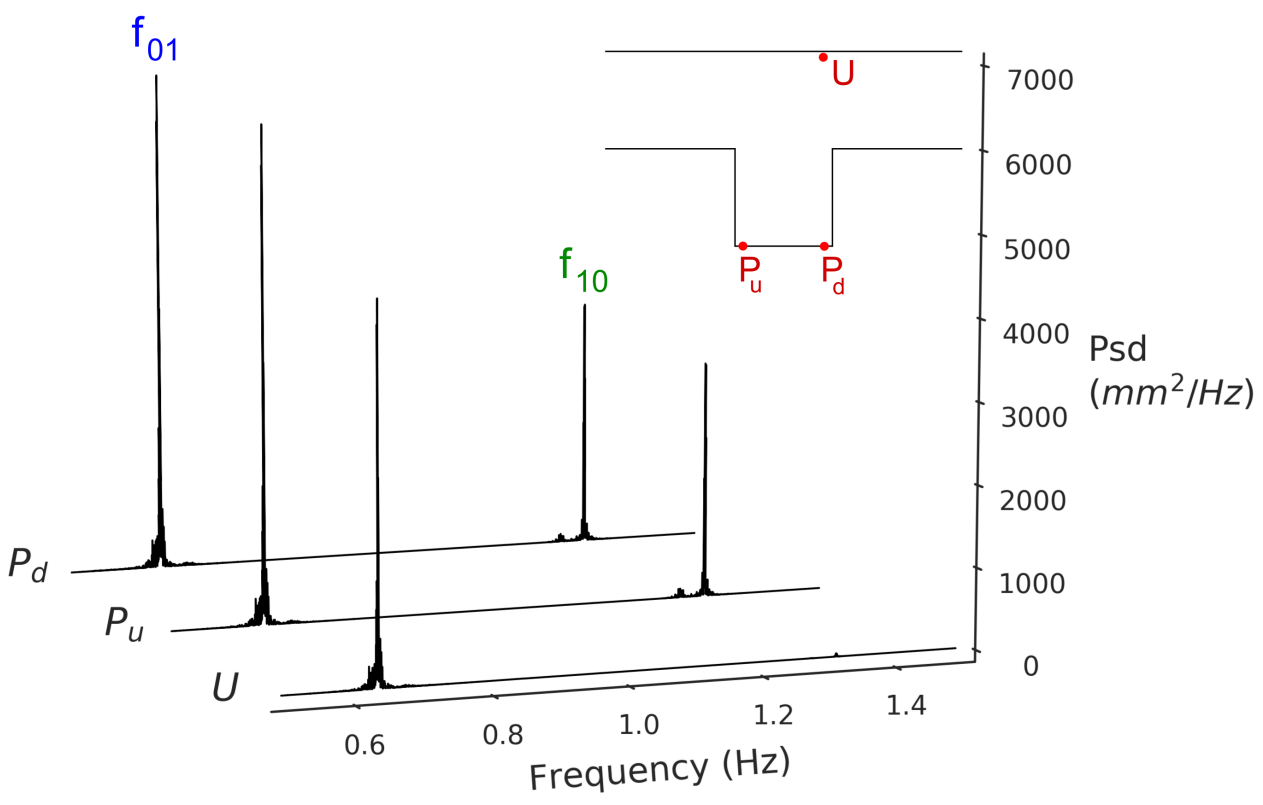

Figure 2. Power spectral density derived by a Fourier transform of the surface registrations recorded by the three measurement devices $\left(P_{u}, P_{d}\right.$ and $\left.U\right)$ for the entire measurement period. The two peak frequencies in the spectra approximately match the theoretically estimated frequencies $\left(f_{01}\right.$ and $\left.f_{10}\right)$ by Equation 1 .

Figure 3 gives the wavelet power spectral density for the time series measured by the upstream pressure sensor, which is analogous to the Psd derived by the Fourier transform and computed as the squared magnitude of the wavelet coefficients $\left|W_{i}\right|^{2}$. Similarly as in Figure 2, the two main characteristic frequencies of the cavity ( $f_{01}$ and $f_{10}$ ) can easily be distinguished as horizontal regions with a high wavelet power. Moreover, Figure 3 shows that seiching is not a stationary phenomenon but is characterised by an intermittent magnitude of the wavelet power at $f_{01}$ and $f_{10}$. Because Figure 2 is based on the integration of the entire surface recordings (90 minutes), it yields a time-averaged power distribution and is thus not representative for the instantaneous power (vertical line in Figure 3) and amplitude of the seiching modes. To derive an estimate for the power (or amplitude) of the seiching waves that is more representative for the physically observed waves, the entire measurement time is subdivided in alternating time intervals in which one of the two seiching directions dominates, i.e. having a larger wavelet power. Two examples of such time windows will be discussed more in detail hereafter and are indicated in Figure 3 by the mode dominating the time window.

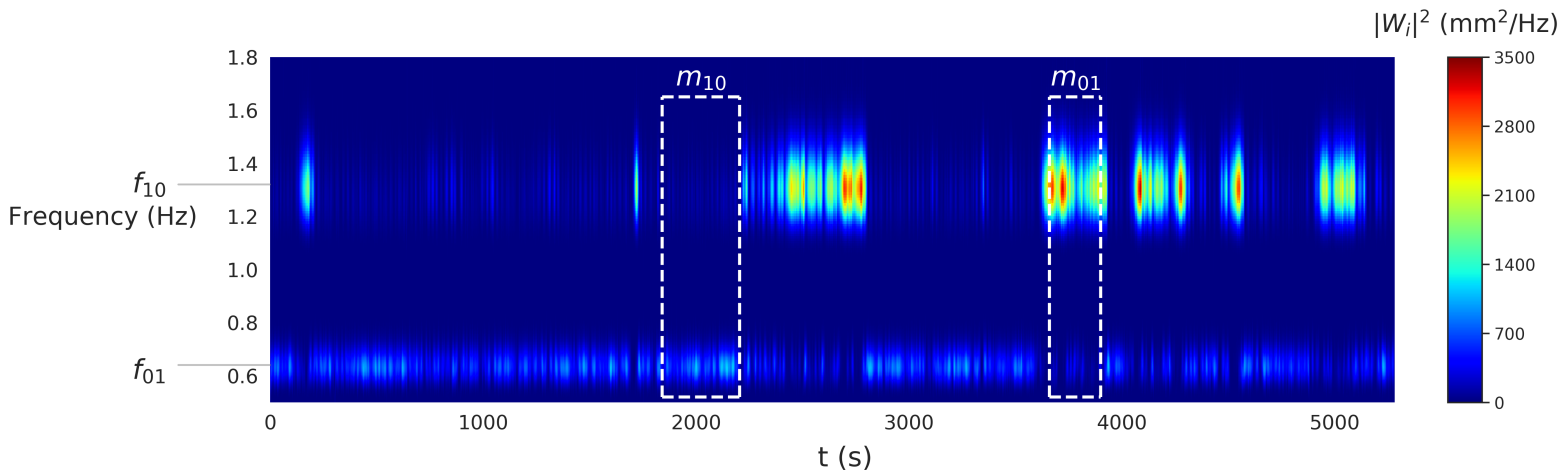

Figure 3. Wavelet power spectrum of the surface recordings of the upstream pressure sensor $\left(P_{u}\right)$ in which the time windows that are analysed in Figure 4 are indicated.

Figure 4 gives the Psd derived by a Fourier transform (similar to Fig. 2) of the surface registrations in the time windows indicated in Figure 3. Figure 4a corresponds to the time window dominated by mode 
$m_{01}$, in which the single, strong peak at $f_{01}$ observed at all three measurement locations confirms that the surface predominantly oscillates in the transverse direction over the combined cavity and main channel width (mode $\left.m_{01}\right)$. For the two pressure sensors $\left(P_{u}\right.$ and $\left.P_{d}\right)$, a small influence of the longitudinal mode $m_{10}$ can be discerned, yet with negligible magnitude.

In contrast, the clear dominant peak frequency at $f_{10}$ in the spectra of $P_{u}$ and $P_{d}$ of Figure $4 \mathrm{~b}$ confirms the dominance of mode $m_{10}$ in the second time window of Figure 3. Similarly as in Figure 2, the absence of the peak at $f_{10}$ for the ultrasonic gauge can be explained by the measurement position $U$ (in the main channel) being located outside the domain of the longitudinal seiching waves (the cavity). Moreover, while still being significantly smaller than the power at $f_{10}$, all three spectra of Figure $4 \mathrm{~b}$ show a non-zero magnitude of the power at $f_{01}$. This bimodal aspect of seiching could already be expected from Figure 3, where the two frequencies sometimes have a non-zero wavelet power simultaneously. However, since even in the short time window of $m_{10}$, of which the length was chosen to achieve a sufficient frequency resolution of the Psd, the wavelet power fluctuates significantly, the concurrency of both seiching modes suggested by Figure $4 \mathrm{~b}$ might be overestimated.
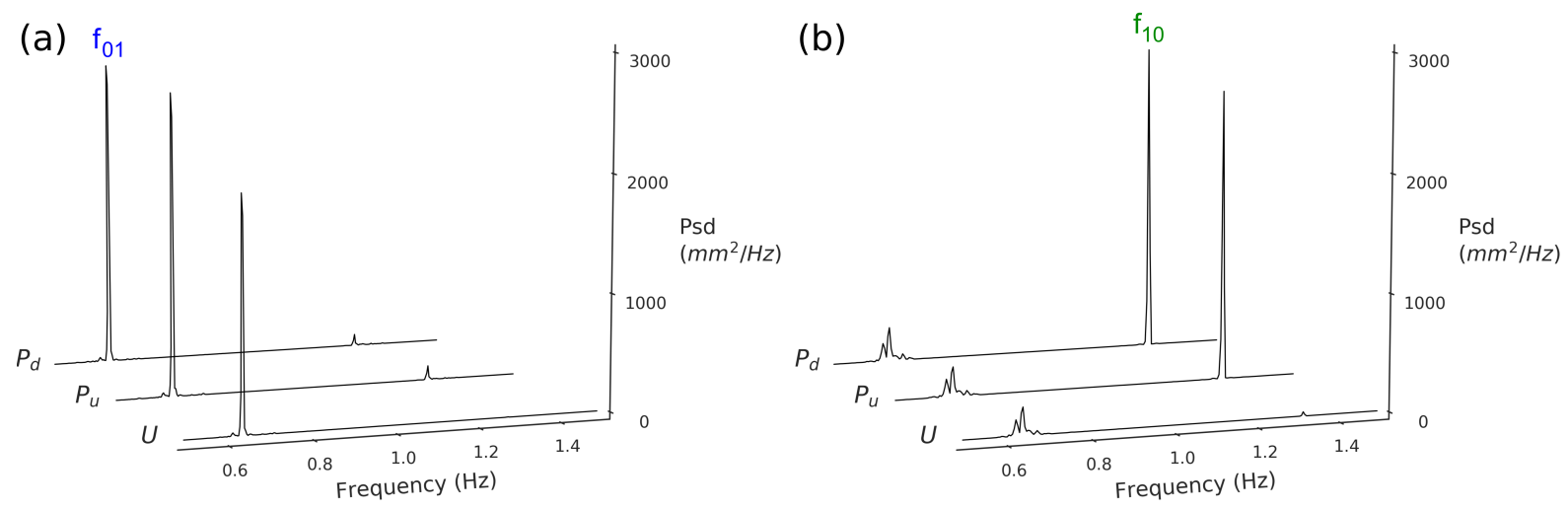

Figure 4. Power spectral density derived by a Fourier transform of the surface registrations recorded by the three measurement devices $\left(P_{u}, P_{d}\right.$ and $\left.U\right)$ for a measurement period in which the surface predominantly oscillates according to the transverse $m_{01}$-mode (a) or the longitudinal $m_{10}$-mode (b).

\section{CONCLUSIONS}

The current contribution has presented an experimental study of the bidirectional seiching waves excited in a lateral, square embayment with a side length equal to the width of the main stream. Due to this particular geometry, the bidirectional aspect of seiching was promoted in the current experiment, for which a Fourier approach was used to compute the (time-averaged) power spectrum of the surface registrations that were measured both inside and outside the cavity area. For the studied flow case, two main seiching modes could be identified, corresponding to a sinusoidal surface shape with a single node in either the transverse or longitudinal direction.

Secondly, a wavelet analysis was applied to investigate the wave power in both the time and frequency domains. The intermittent character of the wavelet power spectrum suggested that bidirectional seiching is not a stationary phenomenon, and showed that the dominant mode of seiching can alternate during the course of a single experiment. Computation of the Fourier power spectrum in well-chosen time intervals revealed that, opposed to what could be concluded from the time-averaged power-frequency distribution, only one of both seiching modes is considerably excited at a single time instance.

On-going research will apply this time-localised strategy to a large number of flow conditions, in which the analysis by Engelen et al. (2018) will be improved to include the intermittent character of bidirectional seiching. Moreover, it is intended that the surface registrations will be combined with simultaneous velocity measurements to quantify other periodic flow features that could be related to the seiching waves. 


\section{REFERENCES}

Akutina, Y. 2015. Experimental investigation of flow structures in a shallow embayment using 3D-PTV. PhD thesis, McGill University, Montreal, QC.

Engelen, L., Creëlle, S., Schindfessel, L., \& De Mulder, T. (2018). Experimental study of the surface oscillations induced by a shallow flow past a lateral cavity. In E3S Web of Conferences, vol. 40, p. 05033.

Faltinsen, O. M., Rognebakke, O. F., \& Timokha, A. N. 2003. Resonant three-dimensional nonlinear sloshing in a square-base basin. Journal of Fluid Mechanics, 487, 1-42.

Kimura, I., \& Hosoda, T. 1997. Fundamental properties of flows in open channels with dead zone. Journal of Hydraulic Engineering, 123(2), 98-107.

Mignot, E., Pozet, M., Rivière, N., \& Chesné, S. 2015. Bidirectional seiching in a rectangular, open channel, lateral cavity. In Congrès français de mécanique. Association Française de Mécanique.

Mignot, E., Cai, W., Launay, G., Rivière, N., \& Escauriaza, C. 2016. Coherent turbulent structures at the mixinginterface of a square open-channel lateral cavity. Physics of Fluids, 28(4), 045104.

Rabinovich, A. B. 2010. Seiches and harbor oscillations. In Kim, Y.C. (ed.), Handbook of Coastal and Ocean Engineering. Singapore: World Scientific.

Teolis, A., \& Benedetto, J. J. 1998. Computational signal processing with wavelets. Boston, MA, USA: Birkhäuser.

Torrence, C., \& Compo, G. P. 1998. A practical guide to wavelet analysis. Bulletin of the American Meteorological society, 79(1), 61-78.

Tsubaki, R., \& Fujita, I. 2006. Surface oscillations in flow past a side cavity using stereoscopic measurement and $P O D$. Journal of hydroscience and hydraulic engineering, 24(2), 41-51.

Tuna, B. A., Tinar, E., \& Rockwell, D. 2013. Shallow flow past a cavity: globally coupled oscillations as a function of depth. Experiments in fluids, 54(8), 1586. 